\title{
Health benefits of probiotics
}

\author{
Barry R. Goldin \\ Department of Family Medicine and Community Health, Tufts University School of Medicine, Boston, MA, USA
}

\begin{abstract}
This paper reviews the evidence for the claims of health benefits derived from the use of probiotics. A brief history of probiotics and the types of probiotics currently used and the criteria for the selection of probiotics is discussed. The ability of probiotics to enhance the nutritional content and bioavailability of nutrients and the scientific evidence for the usefulness of probiotics in alleviating the symptoms of lactose intolerance and in enhancing growth development is examined. The remainder of the review focuses on studies of a specific probiotic, Lactobacillus $G G$ which has been extensively investigated for its health benefits in humans and animals. These studies serve as a model for the potential benefits of probiotics. The ability of Lactobacillus GG to treat or prevent diarrhoeal disease, to serve as an adjuvant for vaccines, to prevent rotavirusinduced diarrhoea, to prevent milk-based allergic reactions, alcohol-induced liver disease and colon cancer are presented. The review concludes with a discussion of the data supporting the safety of probiotics.
\end{abstract}

Probiotics: Health benefits: Lactobacillus GG

For thousands of years microbial cultures have been used to ferment foods and prepare alcoholic beverages. In Genesis references are made to the preparation of fermented milk. In the past century, various different micro-organisms have been tested for their ability to prevent and cure diseases in animals and humans. Micro-organisms have also been added to domestic animal feed to enhance growth. Based on these new applications, the word probiotic was used to describe beneficial micro-organisms by Lilley \& Stillwell (1965). Fuller (1992) defined a probiotic as 'a live microbial feed supplement which beneficially affects the host animal by improving its microbial balance'. In this paper a review of probiotics currently used, selection criteria for probiotics, purported health claims for probiotics and scientific evidence for these claims will be presented.

\section{Results and discussion}

\section{Selection criteria for probiotics}

One criteria which has been used to select probiotics is species compatibility. The general principle is that a probiotic isolated from an animal is less effective in another animal. However, many probiotics are of unknown origin and therefore inter-species use of probiotics is common. The other major selection criteria that have been applied for probiotics include the ability to survive transit through the gastrointestinal tract, adherence to the intestinal epithelial cell lining, production of antimicrobial substances towards pathogens, ability to stabilize the intestinal microflora, antigenotoxic properties, a short generation time, a good shelf life in food or powdered preparations and non-pathogenic properties. In regard to the ability to survive in the gastrointestinal tract a potential probiotic's ability to survive in a low $\mathrm{pH}$ environment and to survive in the presence of bile has been used as a screening technique. Many probiotics currently being used have been assumed to be safe based on past history of a lack of medical problems reported for the genera of which that probiotic is a member. An example has been Lactobacillus and Bifidobacterium. However, there can be species differences in pathogenicity and this is always an issue that should be considered in the selection of probiotics. Animal feeding studies using reasonable doses can be helpful in this regard. In general, probiotics that have been selected in recent years meet at least some of the criteria listed above. In contrast microorganisms that have been used for many years in the food industry and as over-the-counter health supplements often have not met any of the experimental selection criteria listed above and hence often may not survive in the gastrointestinal tract.

\section{Probiotics currently in use}

Table 1 contains a partial list of probiotics that have been used or are currently used for human and animal consumption. The majority of probiotics are bacterial, with the species of Lactobacillus and Bifidobacterium being the most common type of bacteria used (Fuller. 1992).

The list, although appearing relatively long, is actually only a small fraction of all of the micro-organisms known that are believed to be non-pathogenic. Therefore, some specificity has been applied in the past for the selection of probiotics. However, as stated above, the selection process 


\begin{tabular}{l} 
Table 1. Micro-organisms used as \\
probiotics for human and animals \\
\hline Bacteria \\
Lactobacillus \\
acidophilus \\
plantarum \\
casei \\
rhamnosum \\
GG \\
delbrueck subsp. Bulgaricus \\
reuteri \\
fermentum \\
brevis \\
lactis \\
cellobiosus \\
Bifdobacterium \\
bifidum \\
infantis \\
longum \\
thermophilum \\
adolescents \\
animalis \\
Streptococcus \\
lactis \\
cremoris \\
alivarius subsp. thermophilus \\
intermedius \\
Leuconostoc \\
Pediococcus \\
Propionibacterium \\
Bacillus \\
Enterococcus \\
E. faecium \\
Yeast and moulds \\
A. cerevisiae \\
C. pintolopesii \\
A. niger \\
A. oryzue \\
\end{tabular}

has been in large part a result of historical use and not based on scientific criteria.

\section{Probiotics and nutritional enhancement}

Bacterial enzymatic hydrolysis has been shown to enhance the bioavailability of protein and fat (Friend \& Shahani, 1984). Bacterial protease can increase the production of free amino acids which can benefit the nutritional status of the host particularly if the host has a deficiency in endogenous protease production. Lactic acid bacteria have also been shown to increase the content of the vitamin B complex in fermented foods.

In Table 2 the effect of fermentation on folic acid content

Table 2. Folic acid content of unfermented milk and several cultured products

\begin{tabular}{lc}
\hline Product & Folic acid $(\mu \mathrm{g} / 100 \mathrm{ml}$ or $100 \mathrm{~g})$ \\
\hline Milk & $0.13-0.73$ \\
Yoghurt & 3.9 \\
Cottage cheese & $2 \cdot 3-5 \cdot 0$ \\
Sour cream culture & $10 \cdot 8$ \\
Cheddar cheese & $4 \cdot 2$ \\
\hline
\end{tabular}

in various dairy product is shown (Shahani \& Chandan, 1979). Similarly fermentation has been shown to increase niacin in yoghurt, cottage cheese and cheddar cheese and riboflavin in yoghurt (Deeth \& Tomine, 1981).

These studies indicate that to a limited extent the action of micro-organisms either during the preparation of cultured foods or in the digestive tract can either improve the digestibility or absolute amounts of some dietary nutrients.

\section{Probiotics and health benefits}

There is a long list of health and developmental benefits that have been attributed to probiotics (Hitchins \& McDonough, 1989). A partial list of these therapeutic and beneficial claims for probiotics are listed in Table 3 . In this paper no attempt will be made to discuss all of these claims, rather some evidence will be presented for a beneficial role for probiotics in the treatment of lactose intolerance and for weight gain in infants and young animals. The remainder of this review will cover the experimental evidence that has been generated for the beneficial properties of a specific probiotic in the areas of intestinal infection and intestinal immunity, food allergies and colon cancer.

\section{Lactose intolerance}

The disaccharide lactose can cause severe intestinal distress characterized by bloating, flatulence and abdominal pain in individuals with a low level of the intestinal enzyme $\beta$-galactosidase (lactase). This condition severely restricts the use of dairy products. Since the condition tends to become more severe with age, it limits the consumption of calcium-rich foods when they are critically needed due to bone loss in the elderly. During fermentation, lactobacilli produce lactase which hydrolyses the lactose in dairy products to glucose and galactose. During yoghurt fermentation the production of lactase steadily increases. Kim \& Gilliland (1983) found that feeding fermented milk to

Table 3. Health claims for probiotics

Intestinal disorders
Diarrhoea
antibiotic induced
travellers
infantile
Constipation
Colitis
Salmonella and Shigella infections
Lactose intolerance
Flatulence
Other disorders
Vaginitis
Alcohol-induced liver disease
Cancer
Hypercholesteraemia
Other uses
Stabilization of flora
Recolonization of bowel after antibiotic treatment
Treatment of food allergies
Adjuvant for vaccines
Increased weight gain during development (primarily for animals)


lactose-intolerant subjects resulted in a significantly lower level of hydrogen in the breath when compared to the hydrogen levels for subjects fed unfermented milk. Hydrogen in the breath is a marker for bacterial metabolism of lactose in the larger bowel. A lower hydrogen level indicates the lactose was metabolized prior to entering the large intestine. Kolars et al. (1984) found that the administration of $18 \mathrm{~g}$ of lactose in yoghurt resulted in one-third the amount of hydrogen in the breath compared to a similar dose of lactose delivered in milk. Analyses of duodenal aspirates indicated significant lactase activity in the intestine $1 \mathrm{~h}$ after the administration of the yoghurt.

\section{Growth during development}

A number of probiotics have been added to animal feed to increase the weight of domestic animals. The increase in weight gain presumably results from infection control and from increased digestibility of nutrients. Animal feeding studies in young rats (Hargrove \& Alford, 1980; Wong et al. 1983) fed a liquid diet consisting either of milk, yoghurt or milk fermented with $S$. thermophilus have shown a significantly higher weight gain for the animals on the fermented products. In 4 weeks the animals fed milk gained $116 \mathrm{~g} v$. $136.3 \mathrm{~g}$ when fed yoghurt and $131.3 \mathrm{~g}$ when maintained on a $S$. thermophilus fermented milk diet. A similar study has been conducted in humans (Robinson \& Thompson, 1952). Bottle-fed infants during the first month of life gained on average $21.9 \mathrm{ozs}$ when fed a standard formula and $26.5 \mathrm{ozs}$ when $L$. acidophilus was added to the formula.

From a number of different types of observations evidence has emerged that probiotics are beneficial in promoting growth in young animals and humans. The mechanism for this enhanced growth has not been definitively proven and remains speculative and is based on the assumptions stated at the beginning of this section.

\section{Lactobacillus $G G$}

Lactobacillus GG (LGG) is a widely used probiotic which has been investigated in experimental animal models, human experimental studies, clinical human studies, tissue culture and in vitro (Saxelin, 1997). This organism has been more widely studied than any other probiotic over the past 10 years and therefore is a good model for reviewing the potential health benefits of probiotics.

LGG was isolated from a human faecal culture in 1985 (Gorbach, 1996). The organism has been identified taxonomically by using genetic probes and protein electrophoretic patterns as a $L$. rhamnosum. The organism, however, is an atypical rhamnosum because it cannot ferment lactose, maltose or sucrose and only ferments rhamnose very slowly.

\section{Diarrhoeal studies}

An initial report in five patients (Gorbach et al. 1987) indicated that LGG could stop episodes of relapsing diarrhoea caused by a Clostridium difficile produced toxin. The LGG also decreased toxin levels in the faeces of these patients. An additional thirty-two patients have now been studied for the efficacy of LGG in the treatment of relapsing
C. difficile induced diarrhoea (Gorbach, 1996b). Of these thirty-two patients $84 \%$ were cured by initial treatment with LGG daily for 2 weeks. Of the thirty-two patients three were cured upon retreatment with LGG resulting in an overall cure rate of $94 \%$.

LGG has been shown to lower the rate of diarrhoea in Finnish people travelling to Turkey (Oksanen et al. 1990) and Americans travelling to developing countries (Hilton et al. 1997). The study performed with American travellers was a randomized placebo design. The subjects who took the placebo had a $7.4 \%$ per day risk of developing diarrhoea. Those travellers who ingested LGG daily had a $3.9 \%$ per day risk of developing diarrhoea. This translated into a $47 \%$ protection rate which was similar to the $39.5 \%$ protection rate noted for Finnish travellers to one site in Turkey.

LGG has also been shown to be effective for the treatment of children hospitalized for severe diarrhoea. Isolauri $e t$ al. (1991) reported that during the hospitalization all children were rehydrated and realimented. The children were randomized into two groups. One group received a placebo and the second group was given LGG. The duration of diarrhoea was reduced from $2.5 \mathrm{~d}$ for the placebo group to $1.1 \mathrm{~d}(P=0.001)$ for the group given LGG. The number of intestinal immunoglobin-secreting cells (ISC) in the LGG group was almost twice that in the placebo group for immunoglobulin $\mathrm{M}$ (IgM), $\operatorname{IgA}$ and $\operatorname{IgG}$ isotopes.

These studies demonstrate that a probiotic can be effective in treating antibiotic-induced diarrhoea in adults, diarrhoeal disease acquired during travel (which most likely has a mixed bacterial and viral aetiology) and diarrhoeal disease in young children predominantly caused by rotaviruses. Diarrhoea worldwide is a major incapacitating disease in adults and a major cause of death in infants in developing countries. Therefore, the use of probiotics may be an important tool in improving health and nutrition in many developing countries.

\section{Vaccine adjuvant}

In conjunction with studies that indicated LGG increased intestinal immunity in children with rotavirus-induced diarrhoea, Isolauri et al. (1995) tested LGG as an adjuvant to an oral vaccine to rotavirus in children. There was an increase of rotavirus-specific IgM-secreting cells in the LGG group compared to placebo $8 \mathrm{~d}$ postvaccination. LGG also increased $\operatorname{IgA}$ and $\operatorname{IgM}$ seroconversion when measured in paired sera measured prior to vaccination and $30 \mathrm{~d}$ postvaccination.

\section{Food allergy}

The milk protein casein is responsible for the first allergic reactions in milk-fed infants. LGG and other lactobacilli can degrade milk proteins to smaller peptides and amino acids (Sutas et al. 1996). LGG hydrolysis of various different casein proteins decreased the proliferation of mitogeninduced human lymphocytes compared to the non-treated caseins (Sutas et al. 1996). Therefore, the effect of LGG supplementation for infants with atopic eczema was studied. The study was performed at the same time that cow milk 
was eliminated from the diet (Majamaa \& Isolauri, 1997). Thirty-one infants were divided into a placebo group $(n=16)$ who were fed a hydrolysed whey formula and a study group $(n=15)$ who were fed the same formula with LGG added. After 1 month the clinical symptoms alone decreased significantly for the children given LGG $(P=0.004)$ but not for the placebo group. The major improvement in the study group was due to a reduction in the extent and intensity of atopic dermatitis. These results suggest that probiotics may downregulate intestinal inflammation and hypersensitivity reactions in infants with food allergies and resulting atopic eczema.

\section{Liver disease}

Alcohol-induced liver disease has been shown to correlate with plasma levels of bacterial endotoxin. Nanji $e t$ al. (1994) studied the effect of LGG on the level of plasma endotoxin and liver pathology in rats chronically fed alcohol. The concentration of plasma endotoxin was significantly reduced in the group of animals fed LGG. The mean value in the control group was $48.3 \mathrm{pg} / \mathrm{ml}$ and in the group given LGG the plasma endotoxin level was $8.4 \mathrm{pg} / \mathrm{ml}$. There was also a significantly lower $(P<0.01)$ liver pathology score in the LGG-fed group. These results indicate that probiotics may be useful in preventing alcohol-induced liver damage, a common condition in many societies.

\section{Colon cancer}

A dimethylhydrazine (DMH) induced colon cancer model was used in our laboratory (Goldin et al. 1996) to determine if LGG could protect against the formation of colon tumours. If LGG was introduced into the feed 3 weeks prior to initiating a 16-week course of DMH injections (weekly) there was a significant reduction in the incidence of colon tumours $(P=0.012)$ and in the number of tumours per tumour-bearing animal $(P<0.02)$ when compared to animals that were administered DMH but did not receive the LGG supplementation. The magnitude of the tumour reduction was similar to that seen in this model when the dietary fat is drastically reduced. These data suggest probiotics if taken on a regular basis may lower the incidence of colon tumours. However, confirmation of this hypothesis is difficult to obtain in the human population.

\section{Safety of probiotics}

Most probiotics have been designated as 'generally recognized as safe' (GRAS) based on their long history of use in food fermentation. However, there have been occasional reports of bacteraemias and endocarditis associated with Lactobacillus generally in severely immunocompromised individuals. This prompted the suggestion that some type of surveillance be instituted for probiotics. Salminen \& Donahue (1996) reviewed the safety data and found no evidence of probiotics being involved in human infections. This is supported by epidemiological data on the safety of dairy products (Salminen \& Donahue, 1996; Saxelin, 1997). Blood cultures isolated from bacteraemia patients in southern Finland were studied. A total of 5192 blood culture isolates were recovered from patients with bacteraemia. Twelve isolates contained lactobacilli. None of these lactobacilli corresponded to LGG or any other Lactobacillus used in dairy products or pharmaceutical preparations. These data strongly support the fact that Lactobacillus is rarely found in bacteraemial infections and that the current probiotics lack a pathogenic potential. Negative results do not totally rule out the possibility that current or future probiotics can cause infection; however, to date the probiotics currently being used appear to be safe.

\section{Conclusion}

This review article has presented data showing that probiotics have significant potential in improving human health and preventing and treating disease. Future research is required to further delineate this potential and to develop new and improved probiotics.

\section{References}

Deeth HC \& Tomine AY (1981) Yogurt: nutritive and therapeutic aspects. Journal of Food Protection 44, 78-86.

Friend BA \& Shahani KM (1984) Nutritional and therapeutic aspects of lactobacilli. Journal of Applied Nutrition 36, $125-153$.

Fuller R (1992) History and development of probiotics. In Probiotics: the Scientific Basis, pp. 1-7 [R. Fuller ed.]. London: Chapman \& Hall.

Goldin BR, Gualtieri L \& Moore RP (1996) The effect of Lactobacillus $G G$ on the initiation and promotion of dimethylhydrazine-induced intestinal tumors in the rat. Nutrition and Cancer 25, 197-204.

Gorbach SL (1996a) The discovery of Lactobacillus GG. Nutrition Today 31, 2S-4S.

Gorbach SL (1996b) Efficacy of Lactobacillus in treatment of acute diarrhea. Nutrition Todav 31, 19S-23S.

Gorbach SL, Chang TW \& Goldin B (1987) Successful treatment of relapsing Clostridium difficile colitis with Lactobacillus GG. Lancet 2, 1519.

Hargrove RE \& Alford JA (1980) Growth response of weanling rats to heated, aged, fractionated and chemically treated yoghurts. Journal of Dainy Science 63, 1065-1072.

Hilton E, Kolakowski P, Singer C \& Smith M (1997) Efficacy of Lactobacillus $G G$ as a diarrheal preventive in travelers. Journal of Travel Medicine 4, 4I-43.

Hitchins AD \& McDonough FE (1989) Prophylactic and therapeutic aspects of fermented milk. American Journal of Clinical Nutrition 49, 675-684.

Isolauri E, Juntunen M. Routanen T, Sillanaukee P \& Koivula T (1991) A human Lactobacillus strain (Lactobacillus casei sp. strain GG) promotes recovery from acute diarrhea in children. Pediatrics 88, 90-97.

Isolauri E, Joensuu J, Suomalainen $H$, Luomala $M$ \& Vesikari $T$ (1995) Improved immunogenicity of oral Dx RRV reassortant rotavirus vaccine by Lactobacillus casei GG. Vaccine 13, 310-312.

Kim HS \& Gilliland SE (1983) Lactobacillus acidophilus as dietary adjunct for milk to aid lactose digestion in humans. Journal of Dairy Science 66, 959-966.

Kolars JC, Levitt MD, Aouj M \& Savaino DA (1984) Yogurt - an autodigesting source of lactose. New England Journal of Medicine 310, $1-3$. 
Lilly DM \& Stillwell RH (1965) Probiotics: growth promoting factors produced by microorganisms. Science 147, 747-748.

Majamaa H \& Isolauri E (1997) Probiotics: a novel approach in the management of food allergy. Journal of Allergy and Clinical Immunology 99, 179-185.

Nanji AA, Khettry U \& Sadrzadeh SMH (1994) Lactobacillus feeding reduce endotoxemia and severity of experimental alcoholic liver disease. Proceedings of Society of Experimental Medicine and Biology 205, 243-247.

Oksanen PJ, Salminen S, Saxelin M, Hamalainen P, IhantolaVormisto A, Murasniemi-Isovitta L, Nikkari S, Oksonen T, Porsti I, Salminen E, Siitonen S, Stuckey H, Toppila A \& Vapaatola H (1990) Prevention of travellers diarrhea by Lactobacillus GG. Annals of Medicine 22, 53-56.

Robinson EL \& Thompson WL (1952) Effect on weight gain and the addition of Lactobacillus acidophilus to the formula of newborn infants. Journal of Pediatrics 41, 395-398.
Salminen SJ \& Donahue DC (1996) Safety assessment of Lactobacillus strain GG (ATCC 53103). Nutrition Today 31, 32S-34S.

Saxelin M (1997) Lactobacillus GG - a human probiotic strain with thorough clinical documentation. Food Reviews International 13, 293-313.

Shahani KM \& Chandan RC (1979) Nutritional and health aspects of cultured and culture containing dairy foods. Journal of Dairy Science 62, 1685-1694.

Sutas Y, Hurme M \& Isolauri E (1996) Down regulation of anti-CD3 antibody induced IL-4 production by bovine caseins hydrolysed with Lactobacillus $G G$-derived enzymes. Scandinavian Journal of Immunology 43, 687-689.

Wong NP, McDonough FE and Hitchins AD (1983) Contribution of Streptococcus thermophilus to growth-stimulating effect of yogurt on rats. Journal of Dairy Science 66, 444-449. 